EPJ Web of Conferences 84, 06004 (2015)

DOI: $10.1051 /$ epjconf/20158406004

(C) Owned by the authors, published by EDP Sciences, 2015

\title{
(New) molecular ions in the interstellar medium
}

\author{
Evelyne Roueff
}

LUTh Observatoire de Paris, Place J. Janssen, 92190 Meudon, France

\begin{abstract}
We summarize the present knowledge on the molecular ionic content in the interstellar medium and in circumstellar envelopes. Emphasis is given on the most recent detections and the related chemical issues.
\end{abstract}

\section{Introduction}

Despite their low fractional abundances relative to molecular Hydrogen $\left(\sim 10^{-4}-10^{-8}\right.$, corresponding to diffuse and dark regions respectively), molecular ions are an important ingredient of interstellar molecular clouds. They may couple to the ambient magnetic field and allow to slow down the gravitational collapse of star forming regions. We refer the readers to the previous reviews given in this series of conferences on Dissociative Recombination and Applications [1-5] for the description of the major features of interstellar chemistry. Dissociative recombination is a major process in the full chemical network and the main channel of destruction of positive molecular ions when those do not react exothermically with $\mathrm{H}, \mathrm{H}_{2}$. It also contributes to the actual neutral molecular content of the ISM through the different weights of the various possible exothermic channels. One illustrative example is provided by the debate on the branching ratios of $\mathrm{N}_{2} \mathrm{H}^{+}$towards $\mathrm{N}_{2}$ and $\mathrm{NH}$ [6-8], leading to the final agreement that $\mathrm{N}_{2}$ is the major formation product in the DR of $\mathrm{N}_{2} \mathrm{H}^{+}$.

Table 1 displays the presently known ionic content found in the interstellar medium (ISM) and in circumstellar envelopes (CSEs). Both principal and rare isotopic substituted ions are reported. Table 1 also displays the previous mis-identification of $\mathrm{HC}_{4} \mathrm{H}^{+}[9,10]$ by crossed caracters and indicates through question marks two possible candidates for an observed feature corresponding to a rotational constant of $11244 \mathrm{MHz}$ [11] $]^{1}$. Finally, we want to stress that $\mathrm{NH}^{+}$has been searched in the various diffuse regions where the $\mathrm{NH}, \mathrm{NH}_{2}$ hydrides have been detected but that its signature has not been found, despite an available spectroscopy. Molecular ions detected since the last DR conference are displayed in boldface characters and molecular anions are reported in italics. We do not discuss here this class of molecular ions which has been discussed in a specific session of the conference. The present contribution will focus on some recent studies which are still under debate.

\footnotetext{
${ }^{a}$ Corresponding author: evelyne.rouef $f @ o b s p m . f r$

${ }^{1}$ This candidate has further been referred in the literature as B11244.
}

This is an Open Access article distributed under the terms of the Creative Commons Attribution License 4.0, which permits unrestricted use, distribution, and reproduction in any medium, provided the original work is properly cited. 
Table 1. Molecular ions found in the ISM and CSEs.

\begin{tabular}{lllllll}
\hline 2 atoms & 3 atoms & 4 atoms & 5 atoms & 6 atoms & 7 atoms & 9 atoms \\
\hline $\mathrm{CH}^{+}$ & $\mathrm{H}_{3}^{+}$ & $\mathrm{H}_{3} \mathrm{O}^{+}$ & $\mathrm{H}_{2} \mathrm{COH}^{+}$ & $\mathrm{HC}_{3} \mathrm{NH}^{+}$ & $\mathrm{C}_{6} \mathrm{H}^{-}$ & $\mathrm{C}_{8} \mathrm{H}^{-}$ \\
${ }^{13} \mathrm{CH}^{+}$ & $\mathrm{H}_{2} \mathrm{D}^{+}$ & $\mathrm{HOCO}^{+}$ & $\mathbf{N H}_{3} \mathbf{D}^{+}$ & $\mathrm{HC}_{4} \mathrm{H}^{+}$ & & \\
$\mathrm{OH}^{+}$ & $\mathrm{D}_{2} \mathrm{H}^{+}$ & $\mathrm{HCNH}^{+}$ & & & & \\
$\mathbf{S H}^{+}$ & $\mathrm{H}_{2} \mathrm{O}^{+}$ & $\mathbf{C H}_{2} \mathbf{D}^{+}$ & $\mathrm{C}_{4} \mathrm{H}^{-}$ & $\mathrm{C}_{5} \mathrm{H}^{-}$ & & \\
$\mathrm{CO}^{+}$ & $\mathrm{HCO}^{+}$ & $\mathbf{C}_{3} \mathbf{H}^{+}(?)$ & & $\mathrm{C}_{5} \mathrm{~N}^{-}$ & & \\
$\mathrm{SO}^{+}$ & $\mathrm{H}^{13} \mathrm{CO}^{+}$ & & & & \\
$\mathrm{CF}^{+}$ & $\mathrm{DCO}^{+}$ & $\mathrm{C}_{3} \mathrm{~N}^{-}$ & & & \\
$\mathbf{H C l}^{+}$ & $\mathrm{HOC}^{+}$ & $\mathrm{C}_{3} \mathrm{H}^{-}(?)$ & & & \\
& $\mathrm{HCS}^{+}$ & & & & \\
$\mathrm{CN}^{-}$ & $\mathrm{N}_{2} \mathrm{H}^{+}$ & & & & \\
& $\mathrm{N}_{2} \mathrm{D}^{+}$ & & & & \\
& ${ }^{14} \mathrm{~N}^{15} \mathrm{NH}^{+}$ & & & & \\
& ${ }^{15} \mathbf{N}^{14} \mathbf{N H}^{+}$ & & & & \\
& $\mathrm{H}_{2} \mathrm{Cl}^{+}$ & & & & \\
\hline
\end{tabular}

\section{Chlorine chemistry}

With the detection of $\mathrm{HCl}^{+}$in absorption toward the Galactic star-forming regions W31C and W49N by De Luca et al. [12], the amount of detected chlorine containing molecules allows to show a graphical representation where all chlorine containing species have indeed beed detected in the interstellar medium (but not necessarily in the same line of sight). The corresponding chemical schema displayed in Fig. 1, is particularly simple and compact, although DR reaction rate constants of both $\mathrm{HCl}^{+}$and $\mathrm{H}_{2} \mathrm{Cl}^{+}$had not been measured until very recently. The experimental value of the DR rate coefficient of $\mathrm{HCl}^{+}$has been published after the conference [13] and found to be approximated by a fitting function involving 10 parameters, i.e. much more complicated than the usual $A \frac{T}{300} \exp (-\Delta E / T)$ expression. This reflects the detailed physical coupling mechanisms involved in this reaction for which theoretical interpretation is still pending. It should also be stressed that no value has been yet published for the DR rate coefficient of $\mathrm{H}_{2} \mathrm{Cl}^{+}$, despite experiments have been performed at the TSR. In such conditions, full reconsideration of interstellar chlorine chemical modeling appears premature despite the availability of new observations $[14,15]$ of chorine containing species which show indeed that theoretical chemical models are far from successful in interpreting the observations. Despite its apparent simplicity, even atomic chlorine chemistry should be considered with care as ionization energy of $\mathrm{Cl}$ is $1251.2 \mathrm{~kJ} / \mathrm{mole}$, corresponding to $12.967 \mathrm{eV}$, very close from the ionization threshold of atomic Hydrogen where atomic and molecular hydrogen absorption transitions strongly obscure the ambient radiation field. Also, a complete study of recombination of $\mathrm{Cl}^{+}$including radiative channels and dielectric contribution is not available yet.

\section{Tracers of fully symmetric molecular ions}

Fully symmetric molecular species can be observed in absorption through vibrational transitions. A common example is provided by $\mathrm{H}_{3}^{+}$which is currently detected in a large number of lines of sight through infrared absorption of the $v_{1}$ vibrational mode [16]. We do not review this ion which is discussed by Oka in this conference. $\mathrm{CH}_{3}^{+}$has also been searched in various lines of sight where $\mathrm{CH}^{+}$has been found abundant, but only upper limits have been obtained [17]. However, fully symmetric ions escape all possible detections through rotational spectroscopy as no permanent dipole moment is present. $\mathrm{H}_{3}^{+}$contravenes partially that rule as weak rotational transitions may arise from a partial decoupling of nuclear and electronic motions as discussed in [18], giving rise to so called forbidden rotational transitions [19]. However, such a possibility does not hold for heavier molecular ions such as $\mathrm{CH}_{3}^{+}$, $\mathrm{NH}_{4}^{+}$, which is unfortunate, as they play a central role in interstellar chemistry. Replacing one $\mathrm{H}$ nucleus 


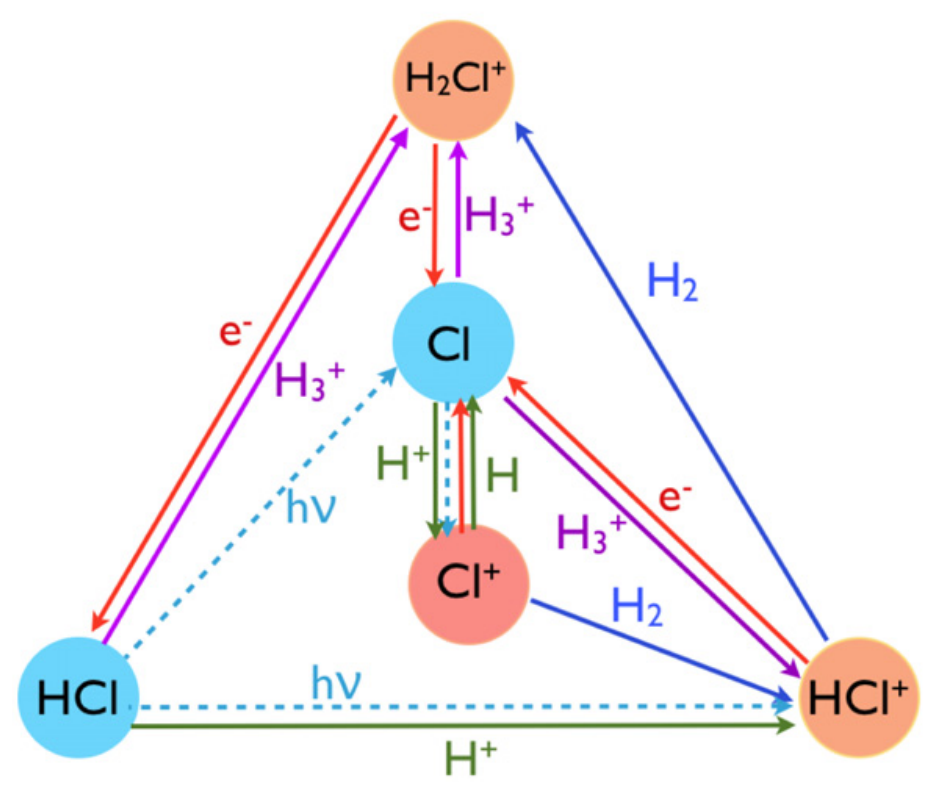

Figure 1. Graphical representation of interstellar chlorine chemistry.

by $\mathrm{D}$ induces a slight asymmetry as the center of mass and the center of charges are no more coincident which results in a permanent (small) dipole moment allowing the corresponding deuterated ion to be studied via rotational spectroscopy. We describe below two recent major advances in this direction.

\section{$3.1 \mathrm{CH}_{2} \mathrm{D}^{+}$}

The $\mathrm{CH}_{2} \mathrm{D}^{+}$molecular ion has been the object of lengthy and difficult observational searches towards different telescopes, both in the $\sim 67 \mathrm{GHz}$ spectral range at the Green Bank Telescope (GBT), 201.8 and $278.7 \mathrm{GHz}$ at IRAM $30 \mathrm{~m}$ telescope and CSO and in a dedicated open time program with Herschel (M. Gerin PI). The astrophysical interest is multiple. First, as mentioned above, the fully hydrogenated corresponding ion $\mathrm{CH}_{3}^{+}$has no rotational spectrum due to its symmetric structure. However, replacement of one $\mathrm{H}$ by $\mathrm{D}$ allows to create some asymmetry and gives rise to a moderate permanent dipole moment estimated to be $0.3 \mathrm{D}$. Then, detecting $\mathrm{CH}_{2} \mathrm{D}^{+}$may allow to deduce the abundance of $\mathrm{CH}_{3}^{+}$which is the starting point of a complex chemistry as pointed out early by [20] and finally better understand the gas phase pathways of molecular complexity. Second, the fractionation reaction between $\mathrm{CH}_{3}^{+}$and $\mathrm{HD}$ leading to $\mathrm{CH}_{2} \mathrm{D}^{+}$, studied in a low temperature ion trap [21], has a significant exothermicity of about $600 \mathrm{~K}$, allowing the reaction to occur at $\sim 50-60 \mathrm{~K}$ in medium warm environments (to the interstellar standards). Third, the spectroscopy of this molecular ion has encountered recent interest both in the Terahertz spectroscopic range [22] and in the infrared [23], where extremely high resolution measurements allow to derive rotational frequencies at a much better accuracy than in previous studies $[24,25]$. Nevertheless, the frequencies available from ground state telescopes have not been directly experimentally studied and a possible unambiguous astrophysical detection would be of great interest. Roueff et al. [26] report observational spectra at the expected 201.8 and $278.7 \mathrm{GHz}$ in the medium warm Orion KL region. Whereas the definitive identification of these spectra with the $\mathrm{CH}_{2} \mathrm{D}^{+}$carrier is not fully secure, due to the limited number of detected frequencies and the possible blend with methylformate at $201.7 \mathrm{GHz}$, and as no experimental rotational spectrum is yet available at these frequencies and the environment is very chemically complex, the claim that the observed spectral 
features come from $\mathrm{CH}_{2} \mathrm{D}^{+}$is plausible and the deduced abundance is also in reasonable agreement with chemical models. The DR reaction rate of $\mathrm{CH}_{3}^{+}$is moderate [27] and has been revisited recently [28] at the heavy storage ring CRYRING. We expect that the corresponding value for $\mathrm{CH}_{2} \mathrm{D}^{+}$is similar but a dedicated study would be important. In addition, the presence of a permanent dipole in $\mathrm{CH}_{2} \mathrm{D}^{+}$ would help to avoid vibrational and rotational excitation which may always persist in such experiments. The DR reaction competes with destruction of $\mathrm{CH}_{3}^{+}$by $\mathrm{H}_{2}$ through radiative association. This latest reaction is slow and found to decrease with increasing temperature [29-34], which reinforces our tentative identification of $\mathrm{CH}_{2} \mathrm{D}^{+}$towards the medium warm Orion KL region.

\section{$3.2 \mathrm{NH}_{3} \mathrm{D}^{+}$}

$\mathrm{NH}_{3} \mathrm{D}^{+}$, deuterated ammonium, is another example of close collaboration between observers, experimental chemists, spectroscopists. The fully hydrogenated ion, $\mathrm{NH}_{4}^{+}$, is the gas phase progenitor of the astrophysical ubiquitous ammonia interstellar molecule, through it dissociative recombination reaction. The channel to ammonia is the most efficient and the corresponding branching ratio is measured to be $70 \%-85 \%$ from experimental studies $[35,36]$. The monodeuterated substitute $\mathrm{NH}_{3} \mathrm{D}^{+}$ has recently been reported as detected via a single rotational transition at $262.8 \mathrm{GHz}$ by Cernicharo et al. [37]. An unknown feature close to the predicted frequency for the $J_{K}=1_{0}-0_{0}$ line of $\mathrm{NH}_{3} \mathrm{D}^{+}$has been first detected in a miraculously void spectral region towards Orion KL. This deuterated ammonium was indeed observed in the infrared in the laboratory by Nakanaga and Amano [38] through its $v_{4}$ vibrational mode from which rotational constants were derived. Higher resolution vibrational spectra allowed to significantly reduce the frequency uncertainties [39] through the same $v_{4}$ vibrational mode and encompassing the detected frequency. The same spectral feature was searched and detected in the $\mathrm{B}_{1}$ cold molecular cloud, as shown on Fig. 2, where abundant deuterated species have been found, including $\mathrm{NH}_{2} \mathrm{D}$ [40] and even ${ }^{15} \mathrm{NH}_{2} \mathrm{D}$ [41]. Unfortunately the other transitions of that molecular ion are not available from ground (from the CDMS database [42]) and the lifetime of Herschel is over. An additional possibility to further constrain the $\mathrm{NH}_{3} \mathrm{D}^{+}$carrier lies in the nuclear hyperfine structure signature. Both nitrogen and deuterium possess quadrupolar momentum and hydrogen atoms have a nuclear magnetic dipole. So, the resulting hyperfine structure is complex. The detected transition is shown to be very narrow in the cold molecular cloud $\mathrm{B} 1$. Then, if the carrier is indeed $\mathrm{NH}_{3} \mathrm{D}^{+}$, the observations induce a constrain on the nuclear coupling constants which should be investigated through theoretical studies. The dissociative recombination reaction rate coefficient of $\mathrm{NH}_{4}^{+}$has been studied theoretically by Douguet et al. [43] in a multichannel quantum defect approach and the isotopic dependence further discussed by Pratt and Jungen [44] for fully deuterated molecular ions. The symmetry breakdown of partial deuteration has to my knowledge not been taken into account in any theoretical study and would be interesting to investigate.

\section{4. $\mathrm{C}_{3} \mathrm{H}^{+}$versus $\mathrm{C}_{3} \mathrm{H}^{-}$?}

In an unbiased deep integration study of the Horsehead nebula environment, Pety and colleagues [11] found several unknown transitions in the so-called PDR (Photon Dominated Region), as shown in Fig. 3, but no corresponding signal was found at the "core" position, corresponding to a cold and dense molecular environment. It was rapidly recognized that these transition frequencies were multiple of a common single factor, $22.48 \mathrm{GHz}$. This fact suggested that a common carrier corresponding to a ${ }^{1} \Sigma^{+}$saturated species was likely and that other transitions could arise for higher rotational quantum numbers at higher frequencies which were available in the spectra. After a successful search of these higher frequency transitions, Pety et al. [11] further suggested that the likely carrier was the $\mathrm{C}_{3} \mathrm{H}^{+}$ molecular ion, regarding the low value of the deduced rotational constant $\mathrm{B} \sim 11.244 \mathrm{GHz}$, close to that of $\mathrm{C}_{3} \mathrm{H}(11.189 \mathrm{GHz})$. The reported value of $\mathrm{B}$ is compatible with ab-initio calculations showing that 

42; 6 B1-BS NH3D+ 30ME2VUI-F02 0:04-FEB-2013 R:04-FEB-2013
RA: 03:33:21.34 DEC: 31:07:26.7 Eq 2000.0 Offs: +0.0 +0.0
Unknown tau: 0.151 Tsys: 249. Time: $3.05 \mathrm{E}+03 \mathrm{~min} \mathrm{El}: 41.4$
$\mathrm{N}: 18688$ I0: $9891.52 \quad$ VO: $6.500 \quad$ Dv: $-0.1114 \quad$ LSR
FO: 262816.730 Df: $9.7656 \mathrm{E}-02$ Fi: 250316.694

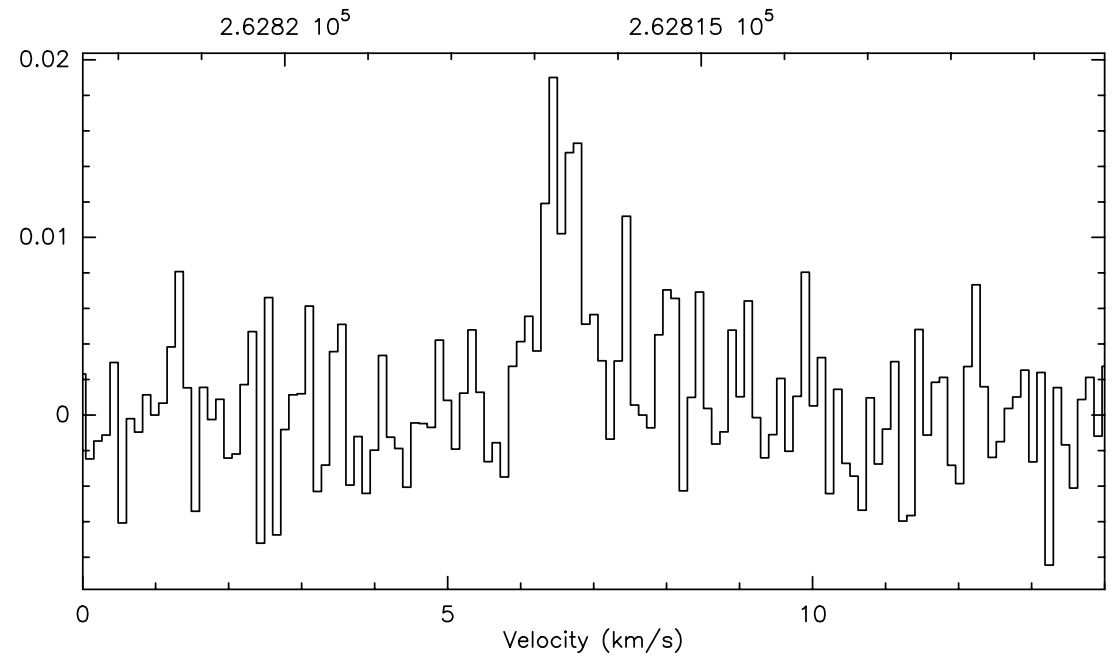

Figure 2. Spectrum of $\mathrm{NH}_{3} \mathrm{D}^{+}$towards B1 taken at IRAM Pico Veleta. The abscissa in $\mathrm{km} / \mathrm{s}$ is linked to the frequency through $\frac{\Delta v}{v}=\frac{v}{c}$. The upper $x$ axis is the frequency in $\mathrm{MHz}$.

1; 5 HORSEHEAD SIGNAL 3MM $\quad-5.0 \quad+0.0$ Eq 135.1
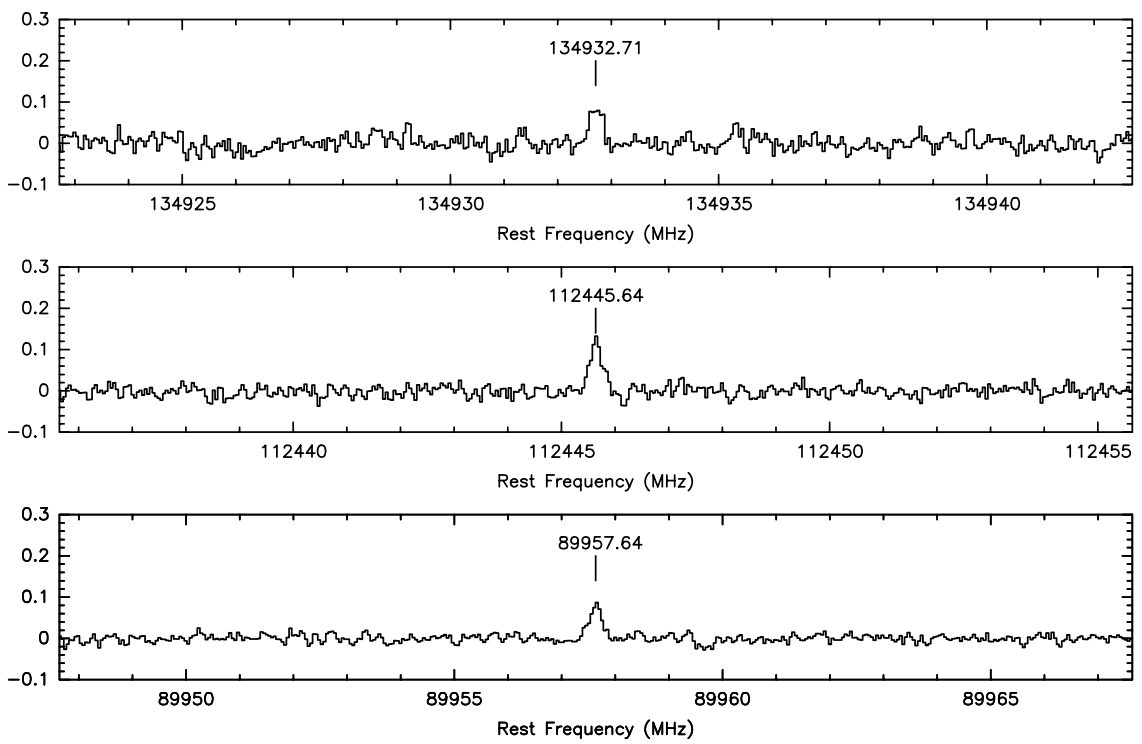

Figure 3. First three unidentified lines detected in the Horsehead nebula. 


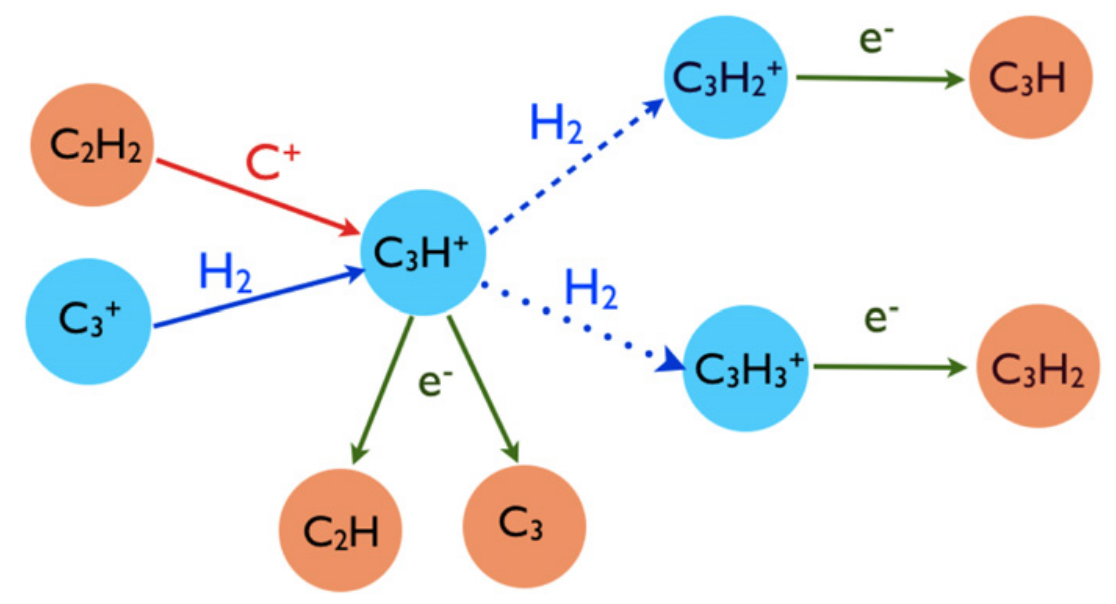

Figure 4. Chemical schema associated to $\mathrm{C}_{3} \mathrm{H}^{+}$. Full (dashed) arrows refer to exothermic (endothermic) bimolecular reactions. the dotted arrow refers to a radiative association reaction.

the most stable geometry of this molecular ion is indeed a closed shell linear form $[45,46]$ and that the corresponding dipole moment is $3.0 \mathrm{D}$. As the high resolution available in heterodyne detectors allows a very precise frequency determination, the spectral analysis achieved in the astrophysical spectra resulted both in the determination of the rotational constant B $(11244.9474 \pm 0.0007 \mathrm{MHz})$ and the centrifugal distortion constant $\mathrm{D}_{e}$, which was found to be $7.766 \pm 0.011 \mathrm{kHz}$.

As far as chemical processes are concerned, the clear spatial dependence of the astrophysical signal allows to emphasize the role of the UV photons, most probably with the atomic ion $\mathrm{C}^{+}$as the starting building block and the subsequent formation of $\mathrm{C}_{2} \mathrm{H}, \mathrm{c}-\mathrm{C}_{3} \mathrm{H}, 1-\mathrm{C}_{3} \mathrm{H}, \mathrm{c}-\mathrm{C}_{3} \mathrm{H}_{2}, 1-\mathrm{C}_{3} \mathrm{H}_{2}$, which were also detected in the same PDR environment. The associated chemical network is displayed on Fig. 4 and represents the main feature of the $\mathrm{C}_{3} \mathrm{H}^{+}$chemistry. Reactions with molecular hydrogen may produce $\mathrm{C}_{3} \mathrm{H}_{2}^{+}$in a slightly endothermic reaction or radiatively associate into $\mathrm{C}_{3} \mathrm{H}_{3}^{+}$and compete with the destruction via dissociative reaction. Then, the ion is predicted to be significantly abundant only in a very restricted area where $\mathrm{C}^{+}$driven chemistry may occur and when molecular hydrogen is not too abundant [11]. We also emphasize that the corresponding experimental spectrum is still not available, although actively searched for since the report of this tentative identification. Aditionally, the transitions expected at lower frequencies in the $80 \mathrm{GHz}$ spectral range were subsequently detected for the first time toward Sgr B2(N) [47] from the publicly available PRIMOS project. The relationship with the neutral $\mathrm{C}_{3} \mathrm{H}$ carbon chain molecule was also emphasized. This identification has been rapidly questioned by theoretical quantum chemists [48] who performed state of the art quantum chemistry calculations of the geometry and electronic structure of $\mathrm{C}_{3} \mathrm{H}^{+}$. They noticed that, whereas the rotational constant is in reasonable agreement with their derivation from the geometry of the cation, the "observational" distortion constant is definitively too large compared to their theoretical value $4.248 \mathrm{kHz}$. Slightly later, the same group further suggested that the carrier of the reported spectral features was rather coming from the molecular anion $\mathrm{C}_{3} \mathrm{H}^{-}$[49], whose ground state is ${ }^{1} A_{1}$, resulting in a slightly asymmetric rotor. Within this hypothesis, the astrophysical transitions detected by Pety et al. [11] would correspond to the $K_{a}=0$ ladder of the representative pseudo symmetric rotor.

The observational clue of that suggestion would require detecting the other possible rotational transitions coming from the molecular anion, i.e. the $K_{a}=1$ ladder which are in a similar spectral range as those involved for $\mathrm{K}_{a}=0$. In a joined effort, astrophysicists have gathered all relevant observed data in the different lines of sight and searched these other possible transitions. However, the values of 


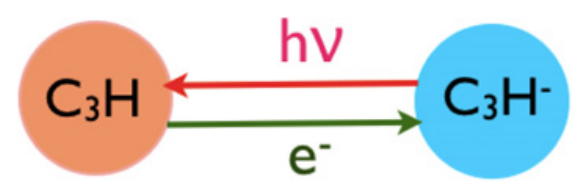

Figure 5. Simple chemical network associated to $\mathrm{C}_{3} \mathrm{H}^{-}$.

the derived frequencies suffer from large error bars, as only $(\mathrm{B}+\mathrm{C}) / 2$ value is constrained from $\mathrm{K}_{a}=0$ transitions. In addition the A rotational constant, which is not very well known has a much larger value of the order of $530 \mathrm{GHz}$, and induces that the lowest energy level with $\mathrm{K}_{a}=1$, corresponding to $\mathrm{J}=1$ is of the order of $\mathrm{A}-(\mathrm{B}+\mathrm{C}) / 2 \sim 518 \mathrm{GHz}$ above the ground $0_{00}$ level, corresponding to an equivalent temperature of about $25 \mathrm{~K}$. Excitation conditions of those $\mathrm{K}_{a}=1$ ladder are thus significantly different from those involved in $\mathrm{K}_{a}=0$. The present situation is that no hint of those transitions has been found in the available spectra [50], which does not necessarily mean that the negative molecular ion is not present. However, assuming that the $K_{a}=0$ ladder transitions of $\mathrm{C}_{3} \mathrm{H}^{-}$are the carrier of the detected spectra, toward the Horsehead nebula, McGuire et al. [50] derive a $\left[\mathrm{C}_{3} \mathrm{H}^{-}\right] /\left[\mathrm{C}_{3} \mathrm{H}\right]$ ratio close to 0.6 , the surprising largest value ever obtained for negative molecular ions. The corresponding ratio of the cation is $\left[\mathrm{C}_{3} \mathrm{H}^{+}\right] /\left[\mathrm{C}_{3} \mathrm{H}\right] \sim 23 \%$, about a factor of 2 smaller than for $\mathrm{C}_{3} \mathrm{H}^{-}$, as less levels are involved in the linear geometric configuration of $\mathrm{C}_{3} \mathrm{H}^{+}$.

Then, we should comment the likelihood of the chemical processes involved in the formation of $\mathrm{C}_{3} \mathrm{H}^{-}$. In the simple hypothesis reported on Fig. 5, that the anion is in equilibrium between associative attachment of $\mathrm{C}_{3} \mathrm{H}$ described by the $\mathrm{k}_{a}$ reaction rate coefficient multiplied by the electronic abundance and the efficient photodetachment probability $k_{\phi}$ of the anion in the $\mathrm{PDR}$, the resulting $\mathrm{C}_{3} \mathrm{H}^{-} / \mathrm{C}_{3} \mathrm{H}$ ratio is

$$
\frac{\left[\mathrm{C}_{3} \mathrm{H}^{-}\right]}{\left[\mathrm{C}_{3} \mathrm{H}\right]}=\frac{k_{a} \times[e]}{k_{\phi}} .
$$

Without trying to include hypothetical numerical values in the various terms, the equation shows clearly that the ratio is maximum when the photodissociation probability is small, which does not correspond to the PDR location but rather to the "Core" conditions. This remark should be tempered by the fact that the neutral carbon chain molecules are only found in the PDR [51], so the final clue on that problem lies in the laboratory attempts. Let us recall that the presence of these carbon chain molecules at the PDR location of the Horsehead nebula is a chemical puzzle as well and that Teyssier et al. [51] suggested they were resulting from the photodissociation of PAHs. As a final remark, we stress out that these transitions have not been found in cold dark molecular clouds such as $\mathrm{B}_{1}$ and special conditions are definitively required to allow the presence of this molecular ion, as no debate is opened on the ionic property of the carrier.

\section{Summary}

We have emphasized some remaining unknown DR reaction rate coefficients. Halogen containing molecular ions are still waiting for quantitative values of DR rate coefficients and corresponding branching ratios. We urge the experimentalists to publish their values which are badly missing for interstellar chemical models. The debate about $\mathrm{C}_{3} \mathrm{H}^{+}$or $\mathrm{C}_{3} \mathrm{H}^{-}$will only be solved after definitive spectroscopic measurements ${ }^{1}$. Meanwhile, chemical processes need to be further constrained: the

\footnotetext{
${ }^{1}$ A novel mass-selective action spectroscopy method based on light induced reactions [52] allowed to obtain the rotational spectrum of $1-\mathrm{CH}_{3}^{+}$and confirm the astrophysical identification by Pety et al. [11].
} 
quantitative value of the total DR of $\mathrm{C}_{3} \mathrm{H}^{+}$is not available until now as well as the radiative attachment rate coefficient of $\mathrm{C}_{3} \mathrm{H}$. Both mechanisms are relevant for the DR community and we sincerely hope that the issue will improve in the following years.

We thank the support of the SUMOSTAI project from the French Agence Nationale de la Recherche.

\section{References}

[1] E. Herbst, Journal of Physics Conference Series 4, 17 (2005)

[2] E. Roueff, Journal of Physics Conference Series 4, 1 (2005)

[3] E. Roueff, E. Herbst, Journal of Physics Conference Series 192, 012008 (2009)

[4] E. Herbst, E. Roueff, Journal of Physics Conference Series 300, 012026 (2011)

[5] E. Roueff, E. Herbst, Journal of Physics Conference Series 300, 012028 (2011)

[6] W.D. Geppert, R. Thomas, J. Semaniak, A. Ehlerding, T.J. Millar, F. Österdahl, M. af Ugglas, N. Djurić, A. Paál, M. Larsson, Astrophys. J. 609, 459 (2004)

[7] C.D. Molek, V. Poterya, N.G. Adams, J.L. McLain, International Journal of Mass Spectrometry 285, 1 (2009)

[8] E. Vigren, V. Zhaunerchyk, M. Hamberg, M. Kaminska, J. Semaniak, M.a. Ugglas, M. Larsson, R.D. Thomas, W.D. Geppert, Astrophys. J. 757, 34 (2012)

[9] J. Krełowski, Y. Beletsky, G.A. Galazutdinov, R. Kołos, M. Gronowski, G. LoCurto, Astrophys. J. Lett. 714, L64 (2010)

[10] J.P. Maier, S. Chakrabarty, F.J. Mazzotti, C.A. Rice, R. Dietsche, G.A.H. Walker, D.A. Bohlender, Astrophys. J. Lett. 729, L20 (2011)

[11] J. Pety, P. Gratier, V. Guzmán, E. Roueff, M. Gerin, J.R. Goicoechea, S. Bardeau, A. Sievers, F. Le Petit, J. Le Bourlot et al., Astron. Astrophys. 548, A68 (2012), 1210.8178

[12] M. De Luca, H. Gupta, D. Neufeld, M. Gerin, D. Teyssier, B.J. Drouin, J.C. Pearson, D.C. Lis, R. Monje, T.G. Phillips et al., Astrophys. J. Lett. 751, L37 (2012)

[13] O. Novotný, A. Becker, H. Buhr, C. Domesle, W. Geppert, M. Grieser, C. Krantz, H. Kreckel, R. Repnow, D. Schwalm et al., Astrophys. J. 777, 54 (2013), 1307. 2995

[14] D.A. Neufeld, E. Roueff, R.L. Snell, D. Lis, A.O. Benz, S. Bruderer, J.H. Black, M. De Luca, M. Gerin, P.F. Goldsmith et al., Astrophys. J. 748, 37 (2012), 1201. 2941

[15] R.R. Monje, D.C. Lis, E. Roueff, M. Gerin, M. De Luca, D.A. Neufeld, B. Godard, T.G. Phillips, Astrophys. J. 767, 81 (2013), 1302.6616

[16] N. Indriolo a, B.J. McCall, Astrophys. J. 745, 91 (2012), 1111.6936

[17] N. Indriolo, T. Oka, T.R. Geballe, B.J. McCall, Astrophys. J. 711, 1338 (2010), 1002.1315

[18] L. Neale, S. Miller, J. Tennyson, Astrophys. J. 464, 516 (1996)

[19] T. Oka, E. Epp, Astrophys. J. 613, 349 (2004)

[20] D. Smith, International Journal of Mass Spectrometry and Ion Processes 129, 1 (1993)

[21] O. Asvany, S. Schlemmer, D. Gerlich, Astrophys. J. 617, 685 (2004)

[22] T. Amano, Astron. Astrophys. 516, L4 (2010)

[23] S. Gärtner, J. Krieg, A. Klemann, O. Asvany, S. Schlemmer, Astron. Astrophys. 516, L3 (2010)

[24] M.F. Jagod, M. Rösslein, C.M. Gabrys, T. Oka, Journal of Molecular Spectroscopy 153, 666 (1992)

[25] M. Roesslein, M.F. Jagod, C.M. Gabrys, T. Oka, Astrophys. J. Lett. 382, L51 (1991)

[26] E. Roueff, M. Gerin, D.C. Lis, A. Wootten, N. Marcelino, J. Cernicharo, B. Tercero, Journal of Physical Chemistry A 117, 9959 (2013), 1306.6795

[27] P.M. Mul, J.B.A. Mitchell, V.S. Dangelo, P. Defrance, J.W. McGowan, H.R. Froelich, Journal of Physics B Atomic Molecular Physics 14, 1353 (1981) 
[28] R.D. Thomas, I. Kashperka, E. Vigren, W.D. Geppert, M. Hamberg, M. Larsson, M.a. Ugglas, V. Zhaunerchyk, N. Indriolo, K. Yagi et al., Astrophys. J. 758, 55 (2012)

[29] D.R. Bates, Phys. Rev. A 34, 1878 (1986)

[30] S.E. Barlow, M. Schauer, G.H. Dunn, Physical Review Letters 52, 902 (1984)

[31] S.E. Barlow, G.H. Dunn, M. Schauer, Physical Review Letters 53, 1610 (1984)

[32] D. Gerlich, G. Kaefer, Astrophys. J. 347, 849 (1989)

[33] M.C. Bacchus-Montabonel, D. Talbi, M. Persico, Journal of Physics B Atomic Molecular Physics 33, 955 (2000)

[34] M.Y. Patuwo, M.B. Lim, R.P.A. Bettens, Chemical Physics Letters 555, 247 (2013)

[35] L. Vikor, A. Al-Khalili, H. Danared, N. Djuric, G.H. Dunn, M. Larsson, A. Le Padellec, S. Rosaen, M. Af Ugglas, Astron. Astrophys. 344, 1027 (1999)

[36] J. Öjekull, P.U. Andersson, M.B. Någård, J.B.C. Pettersson, A.M. Derkatch, A. Neau, S. Rosén, R. Thomas, M. Larsson, F. Österdahl et al., J. Chem. Phys. 120, 7391 (2004)

[37] J. Cernicharo, B. Tercero, A. Fuente, J.L. Domenech, M. Cueto, E. Carrasco, V.J. Herrero, I. Tanarro, N. Marcelino, E. Roueff et al., Astrophys. J. Lett. 771, L10 (2013), 1306. 3364

[38] T. Nakanaga, T. Amano, Canadian Journal of Physics 64, 1356 (1986)

[39] J.L. Doménech, M. Cueto, V.J. Herrero, I. Tanarro, B. Tercero, A. Fuente, J. Cernicharo, Astrophys. J. Lett. 771, L11 (2013), 1306.3792

[40] E. Roueff, D.C. Lis, F.F.S. van der Tak, M. Gerin, P.F. Goldsmith, Astron. Astrophys. 438, 585 (2005), astro-ph/0504445

[41] M. Gerin, N. Marcelino, N. Biver, E. Roueff, L.H. Coudert, M. Elkeurti, D.C. Lis, D. BockeléeMorvan, Astron. Astrophys. 498, L9 (2009), 0903. 3155

[42] H.S.P. Müller, C.P. Endres, J. Stutzki, S. Schlemmer, The CDMS view on molecular data needs of Herschel, SOFIA, and ALMA, in American Institute of Physics Conference Series, edited by J.D. Gillaspy, W.L. Wiese, Y.A. Podpaly (2013), Vol. 1545 of American Institute of Physics Conference Series, pp. 96-109, 1304.1894

[43] N. Douguet, V. Kokoouline, A.E. Orel, Journal of Physics B Atomic Molecular Physics 45, 051001 (2012)

[44] S.T. Pratt, C. Jungen, J. Chem. Phys. 137, 174306 (2012)

[45] D.L. Cooper, S.C. Murphy, Astrophys. J. 333, 482 (1988)

[46] S. Ikuta, J. Chem. Phys. 106, 4536 (1997)

[47] B.A. McGuire, P.B. Carroll, R.A. Loomis, G.A. Blake, J.M. Hollis, F.J. Lovas, P.R. Jewell, A.J. Remijan, Astrophys. J. 774, 56 (2013), 1307.2173

[48] X. Huang, R.C. Fortenberry, T.J. Lee, Astrophys. J. Lett. 768, L25 (2013), 1305. 1658

[49] R.C. Fortenberry, X. Huang, T.D. Crawford, T.J. Lee, Astrophys. J. 772, 39 (2013), 1306. 3963

[50] B.A. McGuire, P.B. Carroll, P. Gratier, V. Guzmán, J. Pety, E. Roueff, M. Gerin, G.A. Blake, A.J. Remijan, Astrophys. J. 783, 36 (2014), 1312. 0153

[51] D. Teyssier, D. Fossé, M. Gerin, J. Pety, A. Abergel, E. Roueff, Astron. Astrophys. 417, 135 (2004), astro-ph/0401309

[52] S. Brünken, L. Kluge, A. Stoffels, O. Asvany, S. Schlemmer, Astrophys. J. Lett. 783, L4 (2014) 